\title{
Ultrawide Bandwidth Wavelength Monitor Based on a Pair of Tilted Fiber Bragg Gratings
}

\author{
Yann Tissot, Hans G. Limberger, and René-Paul Salathé, Senior Member, IEEE
}

\begin{abstract}
A wavelength monitor based on a pair of tilted fiber Bragg gratings is reported. The monitor exhibits a subpicometer wavelength selectivity over a broad wavelength range $(>30 \mathrm{~nm})$, and a low insertion loss $(<1 \mathrm{~dB})$ for laser light with polarization matching the reference polarization of the device. Simulations show that a wavelength coverage up to $100 \mathrm{~nm}$ is possible and that the resolution degradation due to changes in the polarization state can be avoided by proper design of the gratings.
\end{abstract}

Index Terms-Broadband, power monitor, tilted fiber Bragg gratings (TFBGs), wavelength monitor.

\section{INTRODUCTION}

$\mathbf{W}$ ITH increasing complexity of all-optical routing systems, the self-controlled optical network is a promising solution for managing the quality of propagating data signals [1]. The future wavelength-division-multiplexed systems demand narrower channel spacing (typically $<25 \mathrm{GHz}$ ) but also a wider operating bandwidth. At the present, the use of EDFA limits the full potential bandwidth of silica transmission fiber to the $C$ - and $L$-band. The future use of multiple pump Raman amplification pushes the useable wavelength bandwidth to values larger than $100 \mathrm{~nm}$ [2], [3]. Handling these novel networks over a wider coverage of emission wavelength in a robust manner calls for an increase use of performance wavelength monitoring devices. These monitors should isolate a specific cause and location of a signal deterioration. So far, several passive wavelength monitoring techniques have been reported [4]-[6]. However, only an in-fiber wavelength monitor enables us to sense the wavelength that is launched into the fiber network.

Recently, in-line fiber spectrometers based on tilted fiber Bragg gratings (TFBGs) [7]-[10] have been reported. TFBGs have the ability to diffract light out of the fiber core in a dispersive way. The scattered wavelength bandwidth is directly determined by the tilt angle of the grating. An index matching material is used to retrieve light out of the fiber cladding. The scattered light from several wavelength channels is simultaneously detected on single successive detectors [7] or may be focused onto an array of photodiodes [8], [9]. Wavelength selectivities of $7 \mathrm{~nm} \mathrm{[7]} \mathrm{and} 0.1 \mathrm{~nm}$ [8] have been reported, respectively. To achieve a higher wavelength selectivity, devices with more than one TFBG have been proposed. Wielandy et al. reported about a Fourier-transform fiber spectrometer

Manuscript received April 5, 2007; revised July 19, 2007. This work was supported by Intel Corp.

The authors are with the Institute of Imaging and Applied Optics, Advanced Photonics Laboratory, Swiss Federal Institute of Technology, Lausanne (EPFL), CH-1015 Lausanne, Switzerland (e-mail: yann.tissot@a3.epfl.ch).

Color versions of one or more of the figures in this letter are available online at http://ieeexplore.ieee.org.

Digital Object Identifier 10.1109/LPT.2007.905200

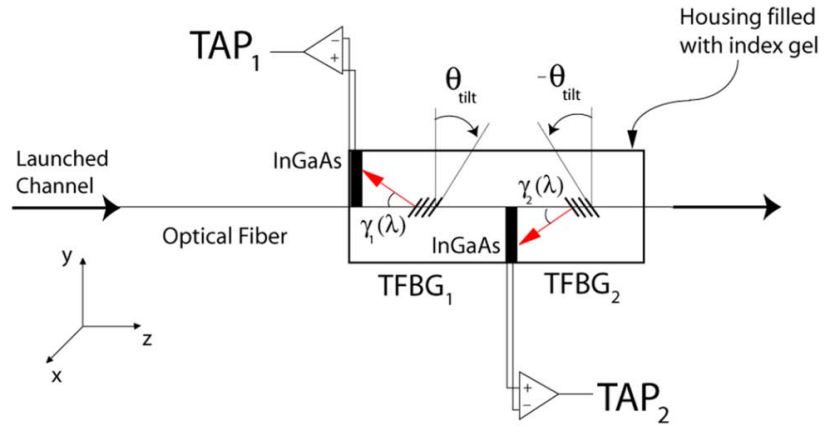

Fig. 1. Scheme of the wavelength monitoring device.

with a maximum wavelength resolution of $\pm 1.5 \mathrm{pm}$ [9] for a 14-mm-long grating. However, the fundamental wavelength resolution of this device is inversely proportional to the length of the fiber Bragg grating (FBG). Mahgerefteh et al. [10], reported about a single wavelength locker fabricated in a polarization-maintaining (PM) fiber. It combines two TFBGs and an FBG that has a strong reflection peak. The new challenge is to design performance wavelength monitors that are compact, inexpensive, easily deployable over the network, and that enable us to cover the entire communication band $\left(L_{-}, C_{-}\right.$, or $S$-band) or even more. In this letter, we propose the use of an identical wavelength monitor for any channel over a wide bandwidth with low loss. Based on the ratio of two TFBG spectra with wavelength dependencies of opposite signs, a wavelength monitor has been realized that has a high selectivity $(<1 \mathrm{pm})$ and a low insertion loss over a communication band of more than $30 \mathrm{~nm}$ [11]. If the polarization state if fixed, the limit in terms of wavelength range and selectivity is given by the signal-to-noise ratio (SNR) of the detection unit. If the polarization state varies, the limits are given by the polarization dependency of the tilted gratings. Simulations show that it is possible to extend the wavelength coverage over $100 \mathrm{~nm}$.

\section{EXPERIMENT}

Two TFBGs have been fabricated in $\mathrm{H}_{2}$-loaded single-mode fibers (SMF-28) using the phase-mask technique and a 244-nm continuous-wave laser source. The fiber gratings have been subsequently annealed for three days at $80{ }^{\circ} \mathrm{C}$ to insure that the $\mathrm{H}_{2}$ molecules have completely diffused out of the fiber and that the gratings are stable over time. Both TFBGs have almost the same insertion loss and full-width at half-maximum (FWHM), but their maxima are separated by more than their FWHM by using two phase masks with different pitch $\left(\Lambda_{1}=1058.6 \mathrm{~nm}\right.$, $\Lambda_{2}=1080.28 \mathrm{~nm}$ ). As illustrated in Fig. 1, the first grating is tilted by an angle of $\theta_{\text {tilt }}=11.5^{\circ}$, whereas the second is tilted by an angle of $\theta_{\text {tilt }}=-11.5^{\circ}$ so that light is scattered 


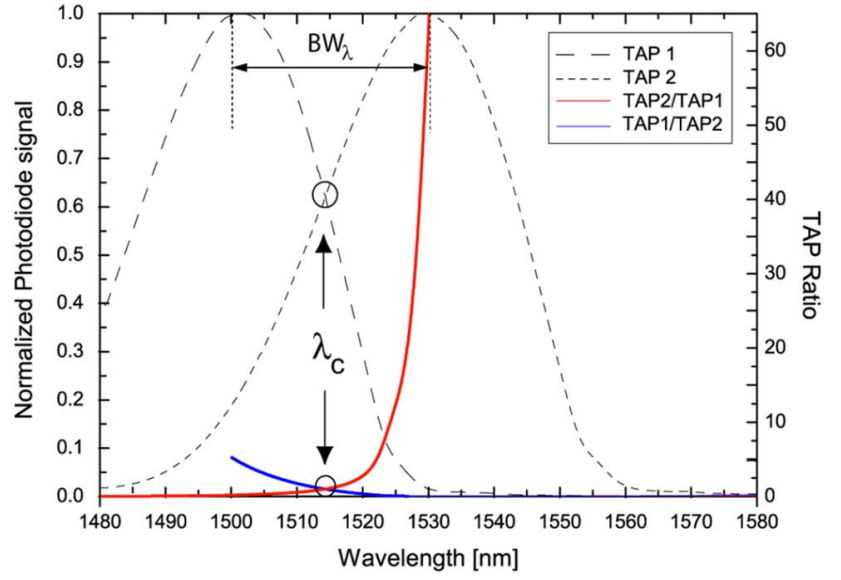

Fig. 2. Measurement of tap spectra and ratio spectra of the wavelength monitor. Solid lines are tap ratios.

in directions symmetric to the fiber axis for high compactness. The gratings have a length of $500 \mu \mathrm{m}$ and an index modulation change of $\delta n=2 \cdot 10^{-3}$. Both gratings are inserted into a housing filled with index matching gel $\left(n=1.444\right.$ at $20^{\circ} \mathrm{C}$ and $1550 \mathrm{~nm}$ ) that contains two InGaAs photodiodes of 1-mm diameter placed perpendicularly to the fiber. Two preamplifiers are used for linearizing the response of the photodiodes. To test the device, a tunable laser (Ando 4321A) with a wavelength stability of $\pm 0.8 \mathrm{pm}$ was employed. Light was launched into the wavelength monitor device by steps of $1 \mathrm{pm}$ over a wavelength range of $100 \mathrm{~nm}$ and measured at the same time using a reference wavemeter with a resolution of $0.1 \mathrm{pm}$ (Burleigh WA-1500). A thermocouple (type $T$ ) was fixed at the module to measure the temperature with a resolution of $\pm 0.001{ }^{\circ} \mathrm{C}$. Light is coupled out by the TFBGs at an angle $\gamma_{i}(\lambda)$ and detected by the photodiodes. The two tap signals and the temperature were monitored over time for each wavelength position in pm-steps.

\section{RESULTS AND DISCUSSION}

Fig. 2 shows the two tap signals as a function of wavelength for an input power of $1 \mathrm{~mW}$ (dashed lines). Both tap signals cross at $\lambda_{c}=1513.160 \mathrm{~nm}$. The maximum insertion loss $(<1 \mathrm{~dB})$ is found at $\lambda_{c}$ and the maximal back reflection is below $-30 \mathrm{~dB}$ and outside the working wavelength band $\mathrm{BW}_{\lambda}$.

Solid curves represent the calculated ratios of both signals that are highly wavelength-selective. In addition, the ratio is independent of laser power fluctuations. This is verified by the measurement of the two tap signals versus the injected power as shown in Fig. 3 for a fixed wavelength $(\lambda=1515.000 \mathrm{~nm})$. Both tap signals are proportional to the laser power launched into the fiber core while the calculated tap ratio is constant. This enables a direct discrimination between power fluctuations and wavelength drift, since in the case of a wavelength change, the tap signal changes are of opposite signs. Hence, the ratio of both tap signals is only wavelength-dependent. It is unity at $\lambda_{c}$ and increases exponentially towards the edges of $\mathrm{BW}_{\lambda}$. The useful wavelength range $\mathrm{BW}_{\lambda}$ is comprised between the two tap maxima which is in this case $1500-1530 \mathrm{~nm}$. The wavelength-selectivity range from $0.6 \mathrm{~dB} / \mathrm{nm}$ at $\lambda_{c}$ to $1.5 \mathrm{~dB} / \mathrm{nm}$ at the maximum of the tap signal.

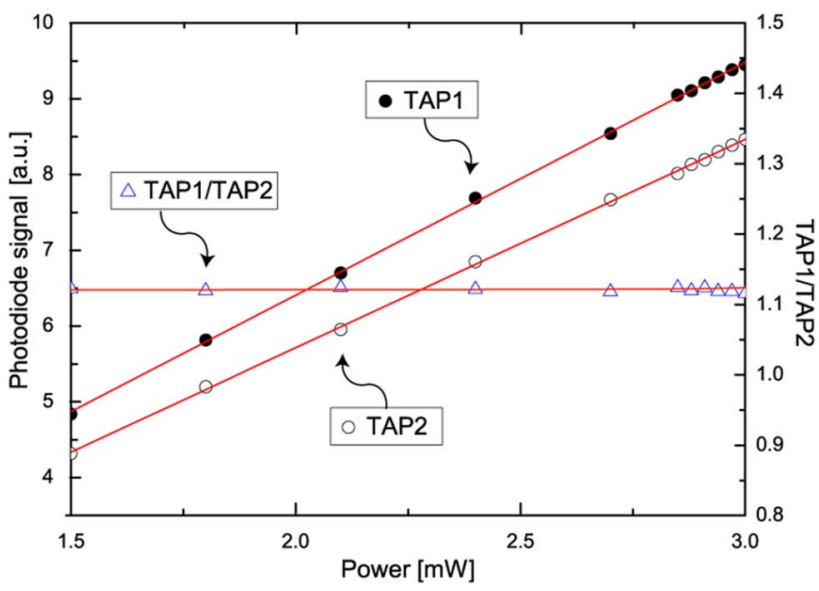

Fig. 3. Measurement of the scattered optical powers and their ratio as a function of the launched laser power at a fixed wavelength $(1515 \mathrm{~nm})$.

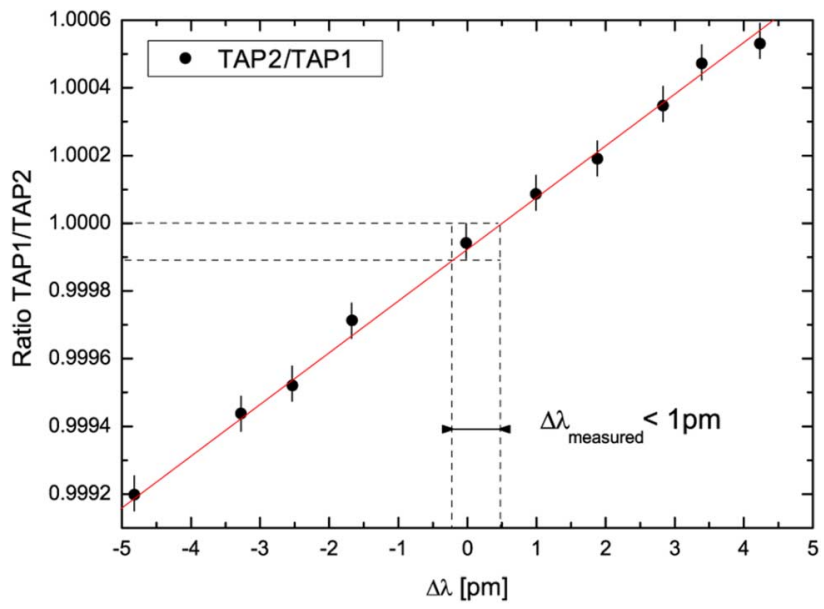

Fig. 4. Measured tap ratio in the vicinity of $\lambda_{c}$, i.e., at the lowest wavelength slope $(0.6 \mathrm{~dB} / \mathrm{nm})$ for a fixed state of polarization.

The performance of the wavelength monitor was evaluated in the vicinity of $\lambda_{c}$, where both tap signals have the same value and the tap ratio varies linearly around unity as illustrated in Fig. 4. The state-of-polarization of the injected signal was fixed and the SNR ratio of the detection system was $36 \mathrm{~dB}$. Each point is an average of 100 measurements taken over $5 \mathrm{~min}$ and error bars represent the calculated variance. The measurements show a wavelength sensitivity at a subpicometer level. The signal deviation can be explained by the temperature fluctuations measured during the experiment $\left( \pm 0.03{ }^{\circ} \mathrm{C}\right)$. As the temperature increases, the wavelength scattered by the gratings shifts accordingly due to the temperature dependency of the silica. The temperature swing typically corresponds to a wavelength shift of $\pm 0.3 \mathrm{pm}$ and thus leads to slightly different tap ratios.

\section{Simulation}

\section{A. SNR Optimization}

Broadening the wavelength bandwidth $\left(\mathrm{BW}_{\lambda}\right)$ can be realized either by increasing the grating pitch difference $\Lambda_{2}-\Lambda_{1}$ or by increasing the grating tilt angle $\theta_{\text {tilt }}$. In order to quantify the maximal wavelength bandwidth the device can cover, simulations have been undertaken for grating pitch differences 


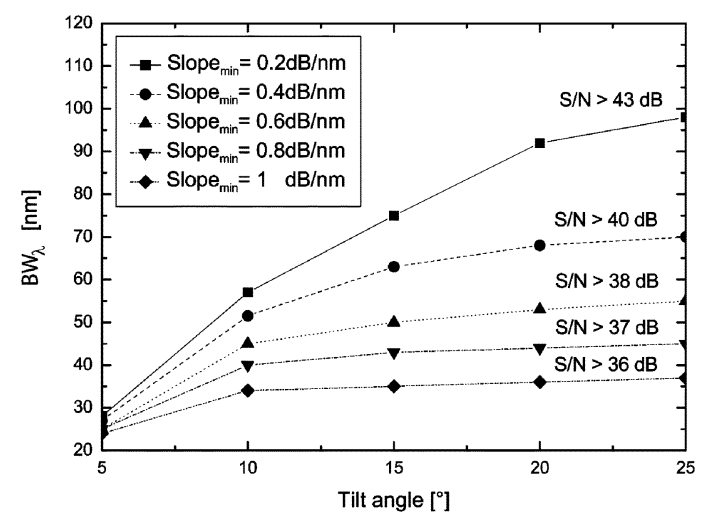

Fig. 5. Simulation of the $\mathrm{BW}_{\lambda}$ as a function of the tilt angle and for different SNR.

ranging from 1 to $80 \mathrm{~nm}$ and pairs of shifted spectra with identical tilt angles $\theta_{\text {tilt }}$ ranging from $5^{\circ}$ to $25^{\circ}$. Twenty-five degrees is the limit for keeping the same device arrangement as illustrated in Fig. 1 with the detector placed perpendicularly to the fiber axis. Spectra have been modeled by the free-space mathematical formalism reported in [12] in the approximation of an infinite cladding that relates to the experimental use of index matching means at the cladding-air interface. Fig. 5 shows the calculated $\mathrm{BW}_{\lambda}$ as a function of the tilt angle for different wavelength selectivities at $\lambda_{c}$. With increasing bandwidth, the wavelength selectivity of the tap ratio is reduced. This can be compensated by a higher SNR.

The $C$-band $(35 \mathrm{~nm})$ can be entirely covered for low tilt angles between $10^{\circ}$ and $15^{\circ}$, with a high wavelength slope $(>1 \mathrm{~dB} / \mathrm{nm})$ and a low SNR $(>36 \mathrm{~dB})$. These specifications correspond to the device characterized in this work. When increasing both the SNR up to $43 \mathrm{~dB}$ and the tilt angle up to $25^{\circ}$, the device presents a promising wavelength coverage close to $100 \mathrm{~nm}$.

\section{B. Polarization Dependency}

To avoid the polarization dependence issue, the devices reported in [7] and [10] have been realized in PM-fibers. As reported by Li et al. [13], tap devices in a standard single-mode fiber show polarization-dependent scattering (PDS). However, the PDS of the ratio of two grating taps $\mathrm{PDS}_{R}$ can be smaller than that of a single tap. It is defined as

$$
\operatorname{PDS}_{R}(\lambda)=\left|\operatorname{PDS}_{1}(\lambda)\right|+\left|\operatorname{PDL}_{1}(\lambda)\right|-\left|\operatorname{PDS}_{2}(\lambda)\right|
$$

with $\operatorname{PDL}_{1}(\lambda)=10 \log _{10}\left(1-\eta_{1}^{p}(\lambda)\right) /\left(1-\eta_{1}^{s}(\lambda)\right)$ where $\eta_{1}^{p, s}(\lambda)$ is the tap efficiency for a linear $p$ - and $s$-polarized laser light and $\operatorname{PDS}_{i}(\lambda)=10 \log _{10}\left(\operatorname{TAP}_{i}^{s}(\lambda) / \operatorname{TAP}_{i}^{p}(\lambda)\right)$. It results from (1) that the polarization dependencies of the first grating in the sequence $\left(\mathrm{PDS}_{1}\right.$ and $\mathrm{PDL}_{1}$ ) partially compensates the PDS of the second grating. Therefore, using a pair of gratings with an identical tilt angle lowers significantly the $\operatorname{PDS}_{R}$. In our case, this reduces the $\mathrm{PDS}_{2}$ from $0.44 \mathrm{~dB}$ to a $\mathrm{PDS}_{R}$ of $0.045 \mathrm{~dB}$ at $\lambda_{c}$. That yields to a minimum wavelength resolution of $75 \mathrm{pm}$ at $\lambda_{c}$. However, it is possible to fine tune this polarization dependency by either increasing the length $L_{g}$ or the index modulation $\delta n$ of the first grating. This increases the polarization-dependent loss (PDL) [14] while the PDS remains the same. Although it remains a technical challenge, it is theoretically possible to cancel the polarization dependence at $\lambda_{c}$ by increasing the length of the first grating from 500 to $620 \mu \mathrm{m}$ for a constant index modulation.

\section{CONCLUSION}

We reported a monitoring scheme based on a highly integrated pair of tilted gratings fabricated in standard telecom fibers. Limitation in terms of wavelength range and wavelength selectivity have been identified and calculated. Temperature controlled, our device combines high wavelength selectivity and a broad wavelength range. Besides, the high integrability of the device can be exploited for high-speed monitoring of the wavelength of a tunable laser over its entire wavelength range.

\section{REFERENCES}

[1] A. E. Willner, "The optical network of the future: Can optical performance monitoring enable automated, intelligent and robust systems?," Opt. Photon. News, vol. 17, no. 3, pp. 30-35, Mar. 2006.

[2] H. Kidorf, K. Rottwitt, M. Nissov, M. Ma, and E. Rabarijaona, "Pump interactions in a 100-nm bandwidth Raman amplifier," IEEE Photon. Technol. Lett., vol. 11, no. 5, pp. 530-532, May 1999.

[3] C. Fludger and V. Handerek, "Ultra-wide bandwidth Raman amplifiers," in Proc. Opt. Fiber Commun. Conf. (OFC), Anaheim, CA, Mar. 2002, pp. 60-62, Paper TuJ3.

[4] R. Giles and S. Jiang, "Fiber-grating sensor for wavelength tracking in single-fiber WDM access PONs," IEEE Photon. Technol. Lett., vol. 9, no. 4, pp. 523-525, Apr. 1997.

[5] Y. Park, S. T. Lee, and C. J. Chae, "A novel wavelength stabilization scheme using a fiber grating for WDM transmission," IEEE Photon. Technol. Lett., vol. 10, no. 10, pp. 1446-1448, Oct. 1998.

[6] H. Nasu, T. Mukaihara, T. Shinagawa, T. Takagi, M. Oike, T. Nomura, and A. Kasukawa, "Wavelength monitor integrated laser modules for 25-Ghz-spacing tunable applications," IEEE J. Sel. Topics Quantum Electron., vol. 11, no. 1, pp. 157-164, Jan./Feb. 2005.

[7] K. S. Feder, P. S. Westbrook, J. Ging, P. I. Reyes, and G. E. Carver, "Infiber spectrometer using tilted fiber gratings," IEEE Photon. Technol. Lett., vol. 15, no. 7, pp. 933-935, Jul. 2003.

[8] J. Wagener, T. A. Strasser, J. Pedrazzani, J. DeMarco, and D. DiGiovanni, "Fiber grating optical spectrum analyzer tap," in Proc. Eur. Conf. Opt. Fibre Commun. (ECOC), 1997, pp. 65-68, Paper PD5.

[9] S. Wielandy and S. C. Dunn, "Tilted superstructure fiber grating used as a Fourier-transform spectrometer," Opt. Lett., vol. 29, no. 14, pp. 1614-1616, 2004.

[10] D. Mahgerefteh, A. M. Benzoni, P. S. Westbrook, K. S. Feder, P. I. Reyes, P. Steinvurzel, B. J. Eggleton, R. G. Ernst, L. A. Reith, and D. M. Gill, "DMRZ: A directly modulated 10-Gb/s RZ source for ultralong-haul WDM systems," IEEE Photon. Technol. Lett., vol. 14, no. 4, pp. 546-548, Apr. 2002.

[11] Y. Tissot, H. Limberger, and R.-P. Salathé, "Low loss wavelength monitor with sub-picometer resolution based on tilted fiber gratings," in Proc. Opt. Fiber Commun. Conf. (OFC) and Exposition and the Nat. Fiber Optic Engineers Conf. (NFOC), Anaheim, CA, Mar. 2007, CD-ROM (OSA, Washington, DC), Paper JThA19.

[12] M. J. Holmes, R. Kashyap, and R. Wyatt, "Physical properties of optical fiber sidetap grating filters: Free-space model," IEEE J. Sel. Topics Quantum Electron., vol. 5, no. 5, pp. 1353-1365, Oct. 1999.

[13] Y. F. Li, S. Wielandy, G. E. Carver, H. L. Durko, and P. Westbrook, "Influence of the longitudinal mode field in grating scattering from weakly guided optical fiber waveguides," Opt. Lett., vol. 29, no. 7, pp. 691-693, 2004.

[14] K. O. Hill, D. C. Johnson, and G. Meltz, "Method and device for reducing polarization dependence in an optical component or optical system," U.S. Patent 6385369 B1, 2002. 